\title{
VIOLÊNCIA SEXUAL CONTRA CRIANÇAS E ADOLESCENTES SOB O OLHAR DAS POSSÍVEIS IMPLICAÇÕES NA VIDA DAS VÍTIMAS: UMA REVISÃO DE LITERATURA
}

\author{
SEXUAL VIOLENCE AGAINST CHILDREN AND ADOLESCENTS \\ THROUGH THE POSSIBLE IMPLICATIONS IN THE VICTIMS' LIVES: A \\ LITERATURE REVIEW
}

\author{
Ana Paula Monteiro do Nascimento ${ }^{1}$ \\ Ana Flávia Henriques Ribeiro Monteiro ${ }^{2}$ \\ Lucas Galvão Araújo ${ }^{3}$ \\ Mateus Louis Rodrigues Cavalcanti ${ }^{4}$ \\ Paloma Medeiros Gomes Cavalcanti ${ }^{5}$ \\ Raissa Cavalcanti Rodrigues ${ }^{6}$
}

RESUMO: OBJETIVO: Este artigo tem como principal objetivo realizar uma discussão a respeito dos impactos da violência sexual em crianças e adolescentes, citar as medidas adotadas pelo governo para reverter essa situação no âmbito da saúde e conhecer o perfil dos agressores tendo como justificativa o crescente número de casos no Brasil. MÉTODO: Utilizou-se a base de dados do PubMed, Scielo, Google acadêmico e documentos fornecidos virtualmente pelo ministério da saúde para estudo e correlação com os dados pesquisados. RESULTADOS: Através dos achados científicos analisados, estima-se que, anualmente, cerca de 225 milhões dos menores são vítimas de abusos sexuais e, destes, a maioria dos casos é do sexo feminino. Os agressores eram homens em mais de $90 \%$ dos casos e os resultados apontam que os pais, padrastos e tios foram os principais responsáveis pelo crime de violência sexual. Ainda há muitos municípios, no país, sem cobertura pelo Programa Sentinela. CONCLUSÃO: Não é possível generalizar ou delimitar com precisão os efeitos do abuso sexual, uma vez que a gravidade e a extensão das consequências são individuais e dependem da experiência de cada

\footnotetext{
${ }^{1}$ Estudante de Medicina do $8^{\circ}$ período do Centro Universitário de João Pessoa - UNIPE.

${ }^{2}$ Estudante de Medicina do $8^{\circ}$ período do Centro Universitário de João Pessoa - UNIPE.

${ }^{3}$ Estudante de Medicina do $8^{\circ}$ período do Centro Universitário de João Pessoa - UNIPE.

${ }^{4}$ Estudante de Medicina do $11^{\circ}$ período do Centro Universitário de João Pessoa - UNIPE.

${ }^{5}$ Estudante de Medicina do $8^{\circ}$ período do Centro Universitário de João Pessoa - UNIPE.

${ }^{6}$ Estudante de Medicina do $5^{\circ}$ período do Centro Universitário de João Pessoa - UNIPE.
} 
vítima. Por isso fica evidente que é preciso uma ação conjunta do Governo com o Sistema de Saúde para detecção precoce, a fim de minimizar danos, possibilitar tratamento e acompanhamento adequados, além de identificar agressores.

Palavras chave: Adolescentes. Criança. Violência sexual.

ABSTRACT: OBJECTIVE: This article has as main objective to carry out a discussion about the impacts of sexual violence on children and adolescents, to mention the measures adopted by the government to reverse this situation in the area of health and to know the profile of the aggressors based on the growing number of cases in Brazil. METHOD: The database of PubMed, Scielo, Google academic was used in addiction to documents provided by the Ministry of Health that were used for study and correlation with the researched data. RESULTS: Through the analyzed scientific findings, it is estimated that, annually, approximately 225 million minors are victims of sexual abuse and, of these, the majority of cases is female. The aggressors were men in more than $90 \%$ of the cases and the results show that parents, stepfathers and uncles were the main responsible for the crime of sexual violence. There are still many municipalities in the country that are not covered by the Sentinela Program. CONCLUSION: It is not possible to generalize or define the effects of sexual abuse perfectly, since the severity and extent of the consequences are individual and depend on the experience of each victim. That is why it is evident that the Government and the Health System must work together for early detection, in order to minimize damage, enable appropriate treatment and monitoring, in addition to identifying the aggressors.

Keywords: Adolescent. Child. Sex offenses. 


\section{INTRODUÇÃO}

A violência acarreta a diminuição da qualidade de vida individual e coletiva constituindo, na sociedade contemporânea, um grave problema de Saúde Pública a nível global. Entre os diferentes tipos de violência, o abuso sexual é uma preocupação constante sendo comum sua ocorrência no ambiente escolar e familiar (SANTOS et al., 2018).

O debate sobre a violência sexual em crianças e adolescentes nos remete à proteção e aos cuidados que esses sujeitos devem receber do Estado, da sociedade, da família, incluindo os profissionais de saúde. Nesse sentido, uma das questões apontadas por alguns estudos é a lacuna do adolescente em ralação a procura por atendimento no setor de saúde, uma vez que eles só buscam o apoio do sistema de saúde, muitas vezes, após a ocorrência de consequências irreversíveis, como uma gravidez indesejada. Diante do exposto, a compreensão da extensão das consequências do abuso sexual infanto-juvenil não é um trabalho fácil, pois existe uma enorme carência na busca desses pacientes por ajuda e de estudos que visem o acompanhamento das consequências físicas, psicológicas e sociais dessas vítimas a longo prazo (TRABBOLD et al., 2016).

Ao discorrer sobre as consequências do abuso sexual praticado contra crianças e adolescentes, é necessário pensar sobre a deturpação da condição física ou biológica que ocorre após o ato. As lesões físicas, decorrentes da violência praticada, demandam, com frequência, vários tratamentos. Os danos psíquicos ocasionados por essa forma de violência interferem no desenvolvimento psicossocial e na estruturação da personalidade da vítima, podendo repercutir ao longo de sua vida. Estudos têm exaustivamente demonstrado que esse tipo de violência sexual ocorre em todos os estratos sociais, não se limita a determinada etnia ou religião, independe de fatores políticos e econômicos e pode atingir crianças e adolescentes de todas as idades dentro e fora do ambiente familiar. Ademais, é visto que a repercussão da violência da vida desses jovens vai além da esfera psicológica, física 
e social e, com isso, podem ocorrer, também, desde fobias até os mais diversos distúrbios sexuais como incapacidade de sentir prazer em relações consentidas (PAIXÃO; DESLANDES, 2010).

Ademais, as violências contra crianças e adolescentes representam grave ameaça aos direitos humanos desse grupo etário e caracterizam-se como uma questão de saúde pública pelo impacto e suas consequências no campo da saúde individual e coletiva, tornando-se uma questão relevante para os profissionais de saúde. Outra questão relevante é que pode resultar em gravidez indesejada, doenças sexualmente transmissíveis e lesões nos órgãos sexuais podendo gerar efeitos de longo prazo e Inter geracionais (SOUTO et al., 2017).

Além disso, as relações sexuais com pessoas com menos de 14 anos são consideradas crime sexual contra vulnerável, de acordo com o Código Penal Brasileiro e isso independe se houve, ou, não o consentimento da vítima, ou, de quaisquer outras circunstâncias (SOUTO et al., 2017). Em relação às consequências psicológicas, a vítima pode desenvolver baixa autoestima, ansiedade, depressão dificuldades sexuais e baixo desempenho escolar podem ser encontradas em jovens com histórico de violência sexual. O impacto dessa violência pode ocorrer, também, sobre seus familiares, nos relacionamentos futuros e no meio social em que vivem essas crianças e adolescentes. Seus efeitos atingem a saúde tanto do indivíduo quanto da sociedade, de forma geral, uma vez que se trata de um fenômeno complexo e de grande magnitude contra indivíduos e isso requer um olhar específico do poder público e uma resposta abrangente (SANTOS et al., 2018).

As situações de abuso sexual enquadram-se na categoria de violência classificada como violência banal o que faz com que, muitas vezes, o agressor saia ileso do crime e desencadeia formas de resistência passiva (LIRA et al., 2017). Destaca-se que apenas em 2009 a notificação de violência passou a fazer parte do Sistema de Informação de Doenças da Declaração Obrigatória (SINAN) o que justifica a escassez de estratégias de intervenção no controle desse problema devido à precariedade do banco de dados. Dessa forma, é visível a necessidade do desenvolvimento dessas estratégias com o objetivo de impedir que novos casos de violência aconteçam ou continuem sendo perpetuados por meio de um ciclo de impunidade e injustiça contra esses jovens (SILVA et al., 2017). 
Nesse contexto, o Plano Nacional foi criado com a finalidade de assegurar a proteção integral às crianças e aos adolescentes em situação ou risco de violência sexual, através de ações organizadas de forma a permitir uma intervenção política, técnica e financeira para o combate à violência sexual infanto-juvenil (PAIXÃO; DESLANDES, 2010). A iniciativa de construir um plano que pudesse nortear as condutas de todos os Estados do país abre as portas para uma mudança, seja ela em tornar público o problema da violência e exploração sexual, seja em mobilizar recursos diversos para seu enfrentamento (SANTOS et al., 2018). Assim, ele tem como metas que dizem respeito ao campo da saúde: criar e articular uma rede de serviços destinada ao atendimento de pessoas em situação de violência sexual; prover atendimento multiprofissional especializado; priorizar a inclusão destas vítimas e seus familiares em programas de saúde; criar programas de intervenção; priorizar o trabalho psicossocial com as famílias e desenvolver programas de formação locais para profissionais e agentes que atuam em programas e instituições de atendimento a situações de violência sexual (PAIXÃO; DESLANDES, 2010).

Em vista disso, ocorreu a implementação do Programa Sentinela em 2001 a fim de atender crianças e adolescentes vitimados pela violência do abuso e pela exploração sexual por meio da criação de Serviços e Centros de Referência (PAIXÃO; DESLANDES, 2010). A criação desse programa como primeiro acontecimento advindo do Plano Nacional refletiu e confirmou o engajamento do governo brasileiro nesta luta. Sua implantação, apesar de extremamente complexa, ocasionou não só uma maior visibilidade sobre a questão do abuso e exploração sexual de crianças e adolescentes, como também potencializou a focalização das ações, a sensibilização e mobilização dos vários setores governamentais e da sociedade civil. Este programa cumpre quase todas as metas do Plano Nacional que visam o "atendimento", com o enfoque ampliado da saúde. Estabelecer uma reflexão crítica do Sentinela e da eficiência de suas ações é uma das formas de analisar este eixo do Plano (SANTOS et al., 2018). 


\section{METODOLOGIA}

Trata-se de uma revisão bibliográfica, qualitativa em que houve a análise de artigos da base de dados do PubMed, Scielo, Google acadêmico e documentos fornecidos virtualmente pelo ministério da saúde relacionados às implicações e consequências do abuso sexual na vida de uma criança ou adolescente.

\section{RESULTADOS E DISCUSSÕES}

Segundo a Organização das Nações Unidas (ONU) mais de $80 \%$ das crianças, no mundo, são castigadas fisicamente, sendo um terço desses castigos considerados muito grave, e, aproximadamente, 50 mil crianças morrem ao ano, em decorrência dessas agressões. De acordo com os dados da ONU, cerca de 225 milhões dos menores são vítimas de abusos sexuais anualmente, destes, a maioria dos casos são do sexo feminino (SENA; SILVA, NETO, 2018). Ainda assim, pesquisadas evidenciaram que, no mundo, cerca de 120 milhões de meninas e jovens com menos de 20 anos de idade já foram forçadas a fazer sexo ou praticar outros atos sexuais (SILVA et al., 2017). No entanto, essa estimativa pode estar subdimensionada devido às circunstâncias em que esses eventos ocorrem, à frequente dependência das vítimas em relação a seu agressor, além do medo e do constrangimento relacionados a dificuldades para denunciar esse tipo de violência. Entre os grupos mais vulneráveis a sofrer violência, encontram-se as crianças e os adolescentes em condição de dependência física, emocional e financeira dos genitores ou responsáveis o que contribui para a pouca visibilidade da real magnitude deste problema, uma vez que grande parte dos casos ocorre no núcleo familiar (SENA; SILVA, NETO, 2018).

Paixão e Deslandes (2010), realizaram uma pesquisa que buscou mapear quem foram os principais agressores e o perfil desses agressores. Os resultados 
apontam que os pais, padrastos e tios foram os principais responsáveis pela crime de violência sexual. Em nenhum dos casos avaliados, mesmo naqueles que se configuraram como extrafamiliares, o agressor era completamente desconhecido para a vítima. Estes dados demonstram que os abusadores muitas vezes utilizam-se da relação de confiança e amizade para cometerem essa violência. De acordo com esses estudos, os agressores eram homens em mais de $90 \%$ dos casos (HABIGZANG; RAMOS, KOLLER, 2011).

Tabela 1 - Frequência e percentagens sobre os agressores.

\begin{tabular}{|c|c|c|c|}
\hline Agressor & $\mathbf{f}^{\mathbf{a}}$ & $\mathbf{\%}$ & \% Casos \\
\hline Pai & 10 & 20,4 & 25,0 \\
\hline Padrasto & 9 & 18,4 & 22,5 \\
\hline Tio & 7 & 14,3 & 17,5 \\
\hline Avô & 4 & 8,2 & 10,0 \\
\hline Irmão & 4 & 8,2 & 10,0 \\
\hline Vizinho & 4 & 8,2 & 10,0 \\
\hline Amigo & 5 & 10,2 & 12,5 \\
\hline Vodastro & 1 & 2,0 & 2,5 \\
\hline Cunhado & 2 & 4,1 & 5,0 \\
\hline Padrinho & 1 & 2,0 & 2,5 \\
\hline Mãe & 1 & 2,0 & 2,5 \\
\hline Madrinha & 1 & 2,0 & 2,5 \\
\hline Total & $\mathbf{4 9}$ & $\mathbf{1 0 0 , 0} \%$ & $\mathbf{1 2 2 , 5 \%}$ \\
\hline
\end{tabular}

Fonte: Elaborada pelos autores. Adaptada de HABIGZANG; RAMOS, KOLLER (2011).

De acordo com Florentino (2015), o debate sobre as consequências do abuso sexual infanto-juvenil leva em conta algumas particularidades, como a violência praticada e o grau de severidade desse ato que varia de acordo com os aspectos individuais do indivíduo que sofre o abuso, tempo de início da violência, idade da criança, o grau de violência utilizado no momento da situação e duração e quantidade de vezes em que ocorreu o abuso.

Dessa forma, segundo Florentino (2014) acontece que, muitas vezes, crianças e adolescentes em situação de vulnerabilidade socioeconômica estão mais expostas a este tipo de violência e suas famílias encontram maiores dificuldades para a superação deste tipo de trauma familiar. Assim, vários são os fatores que 
contribuem para a existência da exploração sexual de crianças e adolescentes. Um dos principais é a violência estrutural expressa na grande desigualdade econômica e social do país. Tal desigualdade é um dos principais fatores que levam à fragilização da rede familiar pela pobreza, violência e outras formas de desestabilização (TRABBOLD et al., 2016). No entanto, é importante dizer que a pobreza não é um determinante deste tipo de situação, uma vez que outros fatores como desemprego, baixa escolaridade, uso de substâncias entorpecentes e histórico de doença mental também representam algumas das causas do abuso sexual infanto-juvenil (CUNHA; SILVA, GIOVANETTI, 2008).

A dificuldade dos profissionais, no que concerne à questão de adolescentes em situação de exploração sexual, nos remete também à complexidade que envolve o tema, uma vez que os casos de abuso sexual são distintos entre si (TRABBOLD et al., 2016). Com isso, de acordo com Florentino (2014), às vítimas têm necessidades singulares de atendimento e acompanhamento o que caracteriza um dos primeiros desafios enfrentados pelos profissionais de saúde que se deparam com tais situações. Dessa forma, esses diferentes padrões, além de dificultarem a padronização das condutas de atendimento e acompanhamento, apontam para a dificuldade por trás da necessidade de compreensão das particularidades de cada caso. Assim, compreender a individualidade de cada paciente é um aspecto fundamental para o planejamento das intervenções de acordo com as necessidades dos envolvidos.

Ademais, Cunha, Silva e Giovanetti (2008) observaram a necessidade de propor um modelo interativo de proteção que contempla às mais diversas abordagens sociais como aspectos fundamentais para a realização de um trabalho multidisciplinar que leve em conta as potencialidades de cada profissional visando uma intervenção mais eficiente no atendimento e acompanhamento das vítimas desse tipo de abuso. Assim, caso haja suspeita ou confirmação de abuso sexual contra crianças e adolescentes é necessário definir a atribuição a ser desempenhada pelos profissionais de cada órgão ou instituição que atua direta ou indiretamente (VECINA; FERRARI, 2002). 
Tabela 2 - População, número de municípios beneficiados pelo sentinela, número de municípios com centros de referência (CR) e capacidade segundo região administrativa do Brasil.

\begin{tabular}{|c|c|c|c|c|}
\hline Região & População & Municípios & Municípios & Capacidade \\
\hline Norte & 13.540 .599 & 449 & 53 & 2.710 \\
\hline Nordeste & 48.845 .112 & 1.792 & 94 & 4.850 \\
\hline Sudeste & 74.447 .456 & 1.668 & 72 & 4.170 \\
\hline Sul & 25.734 .253 & 1.188 & 67 & 3.550 \\
\hline Centro-Oeste & 12.101 .504 & 463 & 45 & 2.590 \\
\hline Brasil & 174.623 .960 & 5.561 & 331 & 17.870 \\
\hline
\end{tabular}

Fonte: Elaborada pelos autores. Adaptada de IBGE e MDS (apud TCU, 2004).

Por meio da análise do gráfico é possível inferir que ainda há muitos municípios sem cobertura do Programa Sentinela o que mantém a violência sexual como uma problemática em grande parte do país.

Ainda assim, segundo Florentino (2014), para que a intervenção realizada junto à criança ou adolescente vítima de abuso sexual ocorra de forma bem sucedida é necessário uma ação integrada entre as instituições e os órgãos de defesa dos direitos da criança e do adolescente, como Sistema Único de Assistência Social (SUAS) e Centro de Referência Especializado de Assistência Social (CREAS). Isso decorre da complexidade desse tipo de violência que, muitas vezes, é encoberto pelos agressores e vítima. Dessa forma, é necessário o envolvimento dos profissionais de saúde para notificar os casos, a fim de que esse problema seja também dimensionado de forma quantitativa e ocorra um planejamento mais voltado às áreas críticas do país e a implementação de políticas e programas de prevenção e intervenção. No Brasil, as informações sobre a dimensão da violência nos serviços de saúde ainda são escassas o que torna maior o desafio de implementação de estratégias para contornar esse contexto (DA-SILVA et al., 2017).

No Brasil, em 2015, os resultados da Pesquisa Nacional de Saúde do Escolar (PeNSE) revelaram que cerca de $4 \%$ dos entrevistados já sofreram o abuso sexual o que foi mais prevalente entre meninas. Segundo dados do Sistema de Informação de Agravos de Notificação (SINAN), a violência sexual ocupa a segunda posição 
entre as agressões contra adolescentes na faixa etária de 10 a 19 anos (MASCARENHAS; RODRIGUES, MONTEIRO, 2018). Além disso, de acordo com a Organização Mundial da Saúde os casos de abuso sexual contra crianças e adolescentes em todos os países do mundo apresentaram uma prevalência maior no sexo feminino (LIRA et al., 2017). No ano de 2015, o SINAN registrou cerca de 162 mil casos de violência contra a mulher, sendo que, desse total cerca de $10 \%$ foram notificações de estupro (SOUTO et al., 2017). Através desses dados é possível analisar que o cenário das relações patriarcais, em que ocorre uma dominação masculina, se configura uma das principais causas desse contexto, uma vez que já se sabe que o perfil dos agressores é predominantemente masculino. Além disso, acentua a submissão e a perpetuação da violência contra mulheres que se encontram em situações em que ocorre um jogo de sedução e ameaça utilizadas pelo agressor para exercer controle sobre a vítima (LIRA et al., 2017).

Com isso, é visível a importância da adoção de padrões de informação, como a construção de um banco de dados em que seja possível quantificar às situações de violência no país, pois permite mensurar a gravidade do problema, emitir informações periódicas atuais e produzir informações confiáveis e oportunas (SILVA et al., 2017). Ademais, o interesse em identificar o abuso sexual é crescente, não somente por suas implicações no processo de integração de crianças e adolescentes à sociedade, mas também pela relação que é possível estabelecer durante a formação da sociedade (MASCARENHAS; RODRIGUES, MONTEIRO, 2018).

\section{CONCLUSÃO}

Conforme foi abordado no presente artigo, as consequências do abuso sexual em crianças e adolescentes são extensas e diversas para as vítimas. Diferentes autores abordam a temática sob os mais singulares pontos de vista. Os demais profissionais de saúde e outras categorias profissionais já tentaram, e continuam tentando, delinear e identificar as consequências decorrentes de uma situação de 
abuso sexual infanto-juvenil, para que, assim, seja possível construir propostas de intervenções mais específicas no sentido de minimizar os danos dessa violência.

Dada à maneira agressiva em que ocorre esse tipo de violência os danos decorrentes da violência sexual constituem um problema de saúde pública. Desta forma, é preciso que os órgãos competentes e os diversos setores em que esses jovens têm algum vínculo trabalhem para a detecção precoce, a fim de interromper sua ocorrência e possibilitar tratamento e acompanhamento adequados de forma precoce a fim de minimizar os danos a essas crianças e adolescentes, além de determinar, também, quem é o agressor daquela vítima e o vínculo que ele tem com ela.

Ainda assim, não é possível generalizar ou delimitar perfeitamente os efeitos do abuso sexual, uma vez que a gravidade e a extensão das consequências são individuais e dependem da experiência de cada vítima. Por isso, é importante que os profissionais de saúde que venham a deparar-se com tais casos tenham a sensibilidade necessária e estejam capacitados para enfrentar essa situação extremamente complexa e desafiadora o que torna a divulgação desta temática e a criação de políticas públicas voltadas para esse assunto tão importantes.

Com isso, é visível que esse tema é um grande desafio para os diversos setores de saúde e para os profissionais que se deparam com sua ocorrência e, assim, é um processo que demanda investimentos na capacitação dos profissionais que lidam, direta ou indiretamente, com crianças e adolescentes, pois a dificuldade na identificação da violência sexual, muitas vezes, está relacionada ao desconhecimento desses profissionais. É necessário, também, que os profissionais de saúde estejam atentos para os sinais e sintomas do abuso sexual entre crianças e adolescentes, visando à detecção e tratamento adequados. 


\section{REFERÊNCIAS BIBLIOGRÁFICAS}

FLORENTINO, Bruno Ricardo Bérgamo. Abuso sexual, crianças e adolescentes: reflexões para o psicólogo que trabalha no creas. Fractal: Revista de Psicologia, [S.L.], v. 26, n. 1, p. 59-70, abr. 2014.

FLORENTINO, Bruno Ricardo Bérgamo. As possíveis consequências do abuso sexual praticado contra crianças e adolescentes. Fractal: Revista de Psicologia, [S.L.], v. 27, n. 2, p. 139-144, ago. 2015.

HABIGZANG, Luísa Fernanda; RAMOS, Michele da Silva; KOLLER, Sílvia Helena. A revelação de abuso sexual: as medidas adotadas pela rede de apoio. Psicologia: Teoria e Pesquisa, [S.L.], v. 27, n. 4, p. 467-473, dez. 2011.

LIRA, Margaret Olinda de Souza Carvalho e; NITSCHKE, Rosane Gonçalves; RODRIGUES, Adriana Diniz; RODRIGUES, Vanda Palmarella; COUTO, Telmara Menezes; DINIZ, Normélia Maria Freire. SURVIVING SEXUAL ABUSE IN EVERYDAY LIFE: forms of resistance used by children and adolescents. Texto \& Contexto - Enfermagem, [S.L.], v. 26, n. 2, p. 327-345, 2017.

PAIXÃO, Ana Cristina Wanderley da; DESLANDES, Suely Ferreira. Análise das políticas públicas de enfrentamento da violência sexual infantojuvenil. Saúde e Sociedade, [S.L.], v. 19, n. 1, p. 114-126, mar. 2010.

SANTOS, Marconi de Jesus; MASCARENHAS, Márcio Dênis Medeiros; RODRIGUES, Malvina Thaís Pacheco; MONTEIRO, Rosane Aparecida. Caracterização da violência sexual contra crianças e adolescentes na escola - Brasil, 2010-2014. Epidemiologia e Serviços de Saúde, [S.L.], v. 27, n. 2, p. 327-345, maio 2018.

SENA, Cláudia Alves de; SILVA, Maria Arleide da; FALBO NETO, Gilliatt Hanois. Incidência de violência sexual em crianças e adolescentes em Recife/Pernambuco no biênio 2012- 2013. Ciência \& Saúde Coletiva, [S.L.], v. 23, n. 5, p. 1591-1599, maio 2018.

SILVA, Lygia Maria Pereira da et al. A escuta de crianças e adolescentes nos processos de crimes sexuais. Ciência \& Saúde Coletiva, [S.L.], v. 18, n. 8, p. 2285-2294, ago. 2013.

SILVA, Priscila Arruda; LUNARDI, Valéria Lerch; LUNARDI, Guilherme Lerch; AREJANO, Ceres Braga; XIMENES, Andrea Stiff; RIBEIRO, Juliane Portella. Violência contra crianças e adolescentes: características dos casos notificados em um centro de referência do sul do brasil. Enfermería Global, [S.L.], v. 16, n. 2, p. 406-466, 28 mar. 2017.

SITUAÇÃO DE VIOLÊNCIA SEXUAL. Psicologia \& Sociedade, [S.L.], v. 28, n. 1, p. 74- 83, abr. 2016.

SOUTO, Rayone Moreira Costa Veloso; PORTO, Denise Lopes; PINTO, Isabella Vitral; VIDOTTI, Carlos Cezar Flores; BARUFALDI, Laura Augusta; FREITAS, Mariana Gonçalves de; SILVA, Marta Maria Alves da; LIMA, Cheila Marina de. Estupro e gravidez de meninas de até 13 anos no Brasil: características e implicações na saúde gestacional, parto e nascimento. Ciência \& Saúde Coletiva, [S.L.], v. 22, n. 9, p. 2909-2918, set. 2017.

TRABBOLD, Vera Lucia Mendes; CALEIRO, Regina Célia Lima; CUNHA, Cristiane de Freitas; GUERRA, Andréa Máris Campo. CONCEPÇÕES SOBRE ADOLESCENTES EM SITUAÇÃO DE VIOLÊNCIA SEXUAL. Psicologia \& Sociedade, [S.L.], v. 28, n. 1, p. 74- 83, abr. 2016. 